\title{
“COMPARATIVE ANALYSIS OF LEGAL ACTS CONCERNING THE PROTECTION OF ANIMALS OF BOVINE AND OVINE SPECIES DURING ROAD TRANSPORT IN THE EUROPEAN UNION AND IN LEBANON" By Julia Havenstein
}

Javier Ernesto Baquero Riveros ${ }^{1}$

En primer lugar, el trabajo de Tesis realizado por Julia Havenstein representa una comparación del estado del bienestar animal durante los procedimientos de transporte de animales de granja, tanto al interior de la Unión Europea, como en los traslados a terceros países, tomando como ejemplo práctico, el caso del Líbano. Allí, se comparan las disposiciones normativas, reglamentos, condiciones y obligaciones que deben cumplirse en esta materia para finalmente poner de relieve la gran diferencia que hay entre el transporte doméstico (que se hace entre estados miembros de la U.E.) y la exportación de especies vivas a terceros países.

Sin embargo, el análisis comparativo no se limita a determinar qué normatividad existe en cada uno de estos territorios y en consecuencia, qué aspectos deben ser mejorados desde el punto de vista legislativo. En efecto, presenta las recomendaciones que en materia de transporte de especies vivas de granja han sido dictadas por la Organización Mundial de Sanidad Animal (O.I.E. - World Organization of Animal Health). Incluso, expone un borrador de proyecto de Ley presentado al Ministerio de Agricultura del Líbano por parte de la asociación no gubernamental "Animals Lebanon", donde se abordan temas de protección y bienestar animal no sólo de animales de compañía, sino de animales de granja y salvajes, con un mayor nivel de protección al existente en la normatividad vigente para el año de publicación de la Tesis (2013). Lamentablemente, el proyecto de Ley no ha sido aprobado por el Parlamento libanés dada la aguda situación política que ha vivido en estos últimos años, lo que desafortunadamente hace que este importante tema pase a un segundo plano.

Como muestra de lo anterior, una realidad preocupante que se evidencia en materia de bienestar animal durante los procedimientos de transporte de animales de granja, mayoritariamente vacunos, cerdos, ovejas y cabras, tanto al interior de la Unión Europea como a terceros países, no es en todos los casos la falta de normatividad, sino su falta de aplicación que, como se verá más adelante, hace que lo escrito muchas veces quede como letra muerta.

A su vez, el estudio revela una triste situación que se puede ver replicada en muchos otros aspectos de la vida diaria en todo el planeta tierra y en aspectos tan variados que nada tienen que ver con el bienestar animal, y ello es que los intereses económicos pasan en muchos casos por encima de las razones éticas y morales. Un

${ }^{1}$ Alumno de la 6⿳a edición del Máster en Derecho Animal y Sociedad de la Universidad Autónoma de Barcelona. 
ISSN: 2462-7518

buen ejemplo de ello y que la autora expone con vehemencia, es que los animales de granja que están destinados a la industria de la carne y por tanto deben ser llevados a mataderos, en vez de ser sacrificados en lugares geográficamente cercanos a donde han sido criados toda su vida, son obligados a soportar el terrible estrés que conlleva una transporte de larga distancia hasta el lugar en donde se les dará muerte, con todos los riesgos que estos procedimientos de larga distancia traen para su salud y bienestar. Esto de por sí genera un riesgo altísimo para su bienestar, pero que sumado a las precarias condiciones en las que son criados en muchos casos, es decir sin las mínimas condiciones de enriquecimiento ambiental, claramente supone un detrimento en su calidad de vida que está motivado únicamente por la reducción de costos de producción.

Así las cosas, dentro de la Unión Europea y cuando los estados miembros realizan un adecuado papel aplicando la normativa comunitaria -enforcement-, es evidente y los resultados así muestran que se puede prevenir gran parte del sufrimiento causado a los animales que sufren el transporte y ojo, digo "prevenir gran parte del sufrimiento", pues al tratarse de una actividad económica legalmente permitida y por costumbre socialmente aceptada, es más que evidente que la cría y el consecuente transporte de animales de granja para las distintas industrias que satisfacen las necesidades del ser humano, no están próximas a acabar. Sin embargo, del estudio también se extrae que cuando los animales son transportados a terceros países, las leyes para mantener y garantizar su bienestar durante este proceso son nulas, o de existir, son absolutamente insuficientes.

En el caso concreto presentado en el estudio, al comparar puntualmente la situación de la Unión Europea con la del Líbano, se puede verificar con lujo de detalles como la situación que les espera a los animales a su llegada al Líbano es, en palabras de su autora, horrenda, pues no sólo se ven expuestos a pobres situaciones de transporte que ponen en gran riesgo su salud física y psicológica, sino que los procedimientos en sí mismos incluyen una alta dosis de crueldad, maltrato y peligro para la vida e integridad de los animales y por si esto fuera poco, terminan en algunos casos con métodos de sacrificio más pertenecientes a épocas medievales, situación que tristemente y desde mi perspectiva, es más común de lo que se cree en regiones apartadas de la Unión Europea e incluso del Líbano, como por ejemplo Sur América.

No es sólo el transporte de largas distancias lo que supone un riesgo para el bienestar de estas especies, pues como bien lo indica la autora, hay una multiplicidad de factores que contribuyen a aumentar la situación de estrés por el transporte, como por ejemplo, las operaciones de carga y desmonte de los animales, que normalmente va acompañada por la inexperiencia, crueldad y salvajismo de las personas responsables de ello, la falta de uso de rampas para permitir el acceso hacia y desde el vehículo, el espacio limitado dentro de los camiones y por tanto el constante roce con los demás animales, la imposibilidad de acceder a los dispensadores de agua y comida, si es que los hay, la imposibilidad de acostarse y levantarse en condiciones normales, el alto riesgo de sufrir resbalones o atascos de 
ISSN: 2462-7518

sus miembros, pezuñas, colas o cuernos, e incluso, el hecho de ser separados de los ejemplares no humanos con quienes han estado acostumbrados a convivir toda su vida y en casos más tristes, sus madres y/o hijos, situaciones que por obvias razones se vuelven mucho más graves cuando se trata de rutas internacionales de transporte, o en cualquier caso, viajes de larga distancia.

No obstante lo anterior, el estudio muestra como al interior de la Unión Europea las políticas sobre bienestar animal están soportadas sobre el principio de las cinco libertades, a saber:

1. "Freedom from hunger and thirst - By ready access to fresh water and a diet to maintain full health and vigour.

2. Freedom from discomfort - By providing an appropriate environment including shelter and a comfortable resting area.

3. Freedom from pain, injury and disease - By prevention or rapid diagnosis and treatment.

4. Freedom to express Normal Behaviour - By providing sufficient space, proper facilities and company of the animal's own kind.

5. Freedom from fear and distress - By ensuring conditions and treatment which avoid mental suffering. ${ }^{2 \prime}$

Lo anterior, se constituye como el espíritu de las distintas disposiciones existentes en la Unión Europea sobre bienestar animal, como es el caso de la Directiva del Consejo de 1977 (77/489/EEC del 18 de julio de 1977), que finalmente fue derogada y sustituida por el Reglamento del Consejo EC 1/2005 sobre la protección de animales durante el transporte, normativa que si bien vela por proteger el bienestar animal, sigue manteniendo el gran problema de la falta de aplicación por parte de los Estados Miembros, al que se hizo referencia anteriormente. $^{3}$

Sin embargo, la ausencia de aplicación de la normativa existente no es el único problema al que se enfrenta la normativa comunitaria. Así, la autora hace especial énfasis en que, si bien existe el Tratado de Funcionamiento de la Unión Europea, el cual en su artículo 13 determina que los animales son (en inglés) "Sentient Beings" y por lo tanto, establece que las exigencias en materia de bienestar animal deben tenerse plenamente en cuenta a la hora de formular políticas de la Unión Europea -colocando el bienestar animal a la altura de muchos otros aspectos de fundamental importancia como por ejemplo la equidad de género-, actualmente no existe una definición oficial (en la U.E.) sobre lo que realmente significa "Sentient Beings", situación que hace que al momento de traducir la expresión a los diferentes

\footnotetext{
2 HAVENSTEIN, Julia. "Protection of animals during transport in the EU and in Lebanon". Deutsche Bibliothek, Deutsche National - Bibliografie. Primera edición, 2014. Página 21.

3 "(...) Enforcement of the Regulation remains a major challenge, partly because of the differences in interpretation of the requirements and because of lack of controls by the Member States. Furthermore, the quality of monitoring data, submitted to the Commission by Member States, is often insufficient to provide a clear analysis of the situation and to allow planning of specific corrective measures al EU level". Ibídem. Pág. 22.
} 
ISSN: 2462-7518

idiomas propios de los Estados Miembros, el espíritu de la misma se vaya desvaneciendo al ser interpretado de una u otra manera, por lo cual, se hace muy complicado alcanzar el verdadero estatus de bienestar animal que fue pensado en la norma.

Por lo anterior, es evidente que al interior de la U.E. existen algunas brechas y/o lagunas de tipo legal que impiden alcanzar el espíritu deseado por la norma comunitaria, pero aún así, salta a la vista que las condiciones establecidas para la protección de los animales de granja durante los procedimientos de transporte dentro de la misma, son mucho más rigurosas y protectoras del bienestar animal que las existentes en terceros países. Así, en el caso puntual tratado por la autora, es evidente como en el caso del Líbano, la situación es a todas luces precaria, pues la normativa que actualmente protege a los animales es la establecida en el Código Penal - Decreto Ley 340 del 01/03/1943, sin que haya sido posible que se apruebe el borrador del proyecto de Ley presentado por "Animals Lebanon" sobre el cual se hizo referencia en líneas precedentes. Por lo tanto, la protección para los animales es prácticamente nula pues la norma antes descrita establece en su artículo 762 que: (en inglés) "(...) Who mistreats or oppresses a domestic animal without any reason will be sentenced to jail or to a monetary fine ranging from 10.000 to 20.000 Lebanese pounds" 4 , de lo cual se pueden extraer varios inconvenientes.

En primer lugar, la norma sólo hace referencia a los animales domésticos, lo cual de por sí puede dar lugar a interpretaciones sobre si los animales de granja o que están bajo el cuidado del ser humano por motivos puramente económicos y de producción, podrían efectivamente ser considerados como domésticos. En segundo lugar, establece que la pena se le impondrá al que incurra en la conducta descrita $\underline{\text { sin }}$ razón alguna, lo que lleva inmediatamente a pensar que existen efectivamente conductas de maltrato animal que estarían permitidas o por lo menos, sobre las cuales podría recaer algún tipo de exclusión de responsabilidad. En tercer lugar y como bien se expone en el libro, actualmente la multa económica establecida en la norma equivale a unos 4,90 EUR, lo cual hace que la sanción sea irrisoria e ínfima en relación con la conducta cometida.

En adición, existen otras disposiciones normativas que de alguna manera regulan los procedimientos de transporte de animales en el Líbano, pero ninguna de ellas lo hace desde una perspectiva de bienestar animal. Por el contrario, son disposiciones en las que se pretende garantizar la salud pública y la sanidad de la comida a través, por ejemplo, de los procedimientos de cuarentena animal, como es el caso del Decreto Ley No. 12301 de 1963, la Decisión No. 263 del 17/10/1940 o la Resolución 829/1 de 2010.

\footnotetext{
${ }^{4}$ Id., pág. 30.
} 
ISSN: 2462-7518

Con todo, el análisis comparativo ${ }^{5}$ de las disposiciones de bienestar y protección animal existentes en la Unión Europea (específicamente el Reglamento del Consejo EC 1/2005), las recomendaciones dictadas por la Organización Mundial de Sanidad Animal (OIE), la normativa existente actualmente en el Líbano y los cambios propuestos en el borrador del Proyecto de Ley, demuestran claramente la necesidad urgente de que los terceros países efectivamente pongan en marcha sus aparatos legislativos para, al menos, cumplir con los estándares mínimos establecidos por la OIE, pues al menos en el caso puntual del Líbano ello no se cumple.

Algunos de los aspectos más llamativos que fueron objeto de comparación, se exponen a continuación. Existen factores que al no estar efectivamente regulados al interior de un país, o incluso estándolo, no son aplicados y en la práctica no se ven sanciones efectivamente impuestas a sus infractores, hacen que las condiciones de bienestar animal de las especies de granja transportadas de un lugar a otro sean absolutamente precarias y deplorables. Entre ellas, se encuentran las terribles aptitudes de los conductores, la falta de espacio existente en los vehículos utilizados para el transporte, lo que normalmente lleva a los animales a golpearse continuamente entre ellos o contra la carrocería del vehículo, a no poder fácilmente acostarse o levantarse por sus propios medios e incluso a no alcanzar los dispensadores de agua y/o comida que deben estar presentes en los vehículos de carga pero que en muchas ocasiones no lo están, la falta de competencia demostrada, además de los conductores, de todos quienes participan en las operaciones previas y posteriores al transporte, como es el caso puntual de los procedimientos de monte y desmonte de animales, en donde por lo general no se utilizan rampas, obligando a los animales a saltar al vacío causándose lesiones fácilmente evitables y a su vez, en donde la falta de entrenamiento, competencia, paciencia, empatía, educación y humanidad de las personas que los realizan, lleva a que se utilice violencia física, verbal y psicológica contra los animales.

A su vez, la aptitud de los animales que serán transportados es objeto de gran debate. En efecto, mientras que en el Reglamento se prohíbe claramente el transporte de animales "severamente comprometidos", lo que es evidente y es el caso de las hembras en su último periodo de gestación o de las crías, incluso establece que en los casos de duda debe existir un veterinario para valorar la aptitud o no del animal para ser transportado; en el caso del Líbano sólo se restringe el transporte de animales que se encuentran enfermos y pueden representar un problema para la salud pública. Por ejemplo, por la contaminación de su carne o por la propagación de algún patógeno a otros animales, pero no se prohíbe el transporte del animal desde la óptica de su propio bienestar y por tanto que esté apto para aguantar el estrés terrible que supone un viaje, en la mayoría de los casos

\footnotetext{
5 Análisis comparativo que la autora centra en 8 ítems a saber: Licencias de las Compañías Transportadoras, Entrenamiento obligado y pruebas de competencia para los conductores y demás personas involucradas en el manejo de los animales antes, durante y después del transporte, Dimensiones Mínimas para el Transporte, Aptitud de los animales para ser transportados, Manejo de los Animales, Tiempo del Viaje, Medios de Transporte y Aplicación de las normas. 
ISSN: 2462-7518

internacional. Por ello, me permito replicar un ejemplo muy evidente del caso, que es expuesto por la autora en la página 53 del libro analizado y que al tenor de las palabras reza: “(...) Thus it would, for example, be possible to transport an otherwise healthy bovine with a fresh open fracture which would cause immense suffering to the animal but would not entail the risk of spreading disease (...)".

De otra parte, el Reglamento establece reglas para determinar la longitud y el tiempo de viaje, pero las mismas no parecen ser tan efectivas. Así, el principio general por el cual se rige el asunto es que (en inglés) "For reasons of animal welfare the transport of animals over long journeys, including animals for slaughter, should be limited as far as possible"6. Por lo cual, el Reglamento determina que la regla general para el transporte de equinos, bovinos, ovinos, caprinos y porcinos no debe exceder de 8 horas, pero lamentablemente esta regla general está llena de excepciones. Así y para citar un ejemplo, en el caso de los bovinos, pueden ser transportados el tiempo que sea, siempre y cuando se realicen ciclos de 29 horas de transporte por 24 horas de descanso y con condiciones específicas de acondicionamiento del vehículo, como por ejemplo, que exista comida y agua suficiente o que la temperatura de los compartimentos donde van los animales este regulada. Lo anterior de ninguna manera cumple el principio general dispuesto por la misma norma, según el cual el transporte debe estar limitado el máximo posible, pues siguiendo estos ciclos podría durar el tiempo que fuera el animal siendo transportado. Ahora bien, si ello parece errado, el caso del Líbano es mucho peor, pues como bien lo expone la autora, no se lograron encontrar requerimientos específicos sobre el tiempo que deben durar los viajes, en la legislación actual libanesa.

De otra parte, sobre la aplicación de la norma o mejor dicho, su falta de aplicación y las diferencias normativas sobre las sanciones existentes al interior de cada país miembro, me permito transcribir un dato sumamente interesante tomado por la autora de un Estudio comparativo sobre la eficacia, proporción y disuasión de las multas monetarias aplicables a los infractores, publicado por las organizaciones World Society for Protection of Animals (WSPA) y Dutch Animal Welfare Organization "Eyes on Animals", que al tenor de las palabras dice: “(..) a Dutch pig truck with an exceded loading density would be fined 2.500 EUR if it were stopped in Holland. If the same truck were stopped in Belgium, the transporters would most likely not be fined since the Belgium authorities cannot chase foreigners to pay open fines. In France the same truck would be fined 135 EUR and if stopped in Italy it would be fined 1.333 EUR (...)"7. Así, es evidente que las diferencias internas sobre aplicación de la norma, así como la multiplicidad y variabilidad de sanciones, ponen en riesgo el bienestar de los animales transportados al no existir coherencia y uniformidad sobre las sanciones aplicables a los infractores de la norma comunitaria.

\footnotetext{
${ }^{6}$ HAVENSTEIN, Julia. "Protection of animals during transport in the EU and in Lebanon". Deutsche Bibliothek, Deutsche National - Bibliografie. Primera edición, 2014. Página 59.

${ }^{7}$ Ibídem. Pág. 68. 
ISSN: 2462-7518

En conclusión, el análisis muestra que aún falta mucho camino por recorrer si queremos lograr un estado real de protección y bienestar durante el transporte de animales de granja. Sobretodo en el caso de las exportaciones a terceros países e incluso en el caso de transportes "domésticos", donde debe haber un mayor compromiso por parte de los Estados Miembros para aplicar efectivamente la normativa comunitaria y modificar, en los casos necesarios, las normativas internas con el fin de generar congruencia y uniformidad en las sanciones aplicadas a los infractores. Esto resulta extraño, pues comúnmente el bienestar animal se observa desde una perspectiva antropocéntrica, lo que implica que supuestamente se deban mejorar las condiciones de bienestar para así aumentar la calidad de la producción, pero incluso bajo esa óptica, el tema se ve relegado en las agendas legislativas de muchos países.

Finalmente, quisiera resaltar la dedicatoria que la autora hace de su Tesis a Antoine, un joven Toro Francés que murió al no soportar el tremendo estrés que implicaba su transporte a Marruecos y así, felicitar el trabajo investigativo realizado con una alta dosis de profesionalismo, lo cual no es muy fácil de lograr cuando se investiga, escribe, denuncia y/o trabaja por defender, mantener o conquistar algún peldaño de bienestar y respeto hacia los animales en campos como este, donde es tan evidente la falta de humanidad de algunos "seres humanos". 